\title{
Symphysis pubis dysfunction in pregnancy-fact or fantasy?
}

\author{
Papa Essilfie $^{1^{*}}$, M. Hussain ${ }^{2}$ \\ ${ }^{1}$ Wirral University Teaching Hospital, UK \\ ${ }^{2}$ St Michaels Hospital, Bristol, UK \\ Email: “jingo78@aol.com
}

Received 29 September 2012; revised 30 October 2012; accepted 8 November 2012

\begin{abstract}
Symphysis pubis dysfunction (SPD) has been recognized as an Obstetric condition since the time of Hippocrates. In spite of this long history, many medical staff remain unaware of the condition. Women continue to suffer from symphysis pubis pain with considerable reduction in quality of life during pregnancy. In recent years there has been an upsurge in the reported incidence of SPD partly due to gross previous under recognition. The increase in incidence has also been attributed to the sudden discovery of the SPD phenomenon by some women who have no symptoms but are generally fed up with being pregnant. The allegation has been that these women use SPD as a reason to request for induction of labour.
\end{abstract}

Keywords: Symphisis Pubis Pain; Ostetis Pubis; Trendelenbug Sign

\section{INTRODUCTION}

The symphysis pubis is a non-synovial cartilaginous joint. The stability of this joint is integral to the function of the pelvis. SPD occurs when the symphysis pubis is unable to effectively perform its role in pelvic stabilization. The wide variation in the incidence of SPD quoted in the literature is due in part to the various names given to this condition. SPD has been referred to as pelvic girdle relaxation, peripartum pelvic pain and Symphysis diastases by some authors. There is an urgent need for standardisation of terminology in reference to pubic dysfunction so that appropriate management guidelines can be made.

\section{PATHOLOGY}

The atieology of Symphysis pubis pain remains unknown. It is however well known that during pregnancy there is relaxation and weakness of pelvic ligaments and joints under the influence of maternal hormones (mainly relaxin). Pelvic ligament weakness leads to instability in the

\footnotetext{
*Corresponding author.
}

pelvic joints. This instability may be sufficient to cause the movement of the bone ends at the symphysis pubis thus stimulating mechanoreceptors with a nociceptive function and resulting in pain. The articular surfaces of the pubic joint are lined with a thin layer of hyaline cartilage and joined by a fibrocartilaginous disc resulting in an average gap of about $5 \mathrm{~mm}$ between the pubic bodies [1]. This interpubic gap increases by an average of about $3 \mathrm{~mm}$ by the third trimester of pregnancy returning back to non-pregnant dimensions by 3 to 6 months after delivery. A pubic gap of more than $10 \mathrm{~mm}$ is often quoted as being abnormal [2]. There has been suggestions that it is at this threshold that symptoms of SPD appear. The relationship between the symphyseal gap and symptoms of SPD still remain controversial. Several studies have shown no correlation between the degree of symphyseal separation and the severity of symptoms.

The role of relaxin in the atielogy of SPD has been heavily contested. This hormone is produced primarily in the corpus luteum. Peak levels of this hormone occur from early to mid pregnancy. Levels drop off rapidly within hours of delivery with a return to normal levels by 4 - 12 weeks postpartum. In conjunction with oestrogen and progesterone, relaxin causes collagenolysis and remodelling of ligamentous tissue. It has been suggested that this re-modelling results in ligamentous extensibility thus starting the process of instability at the pubic joint. Some studies have shown a high level of serum relaxin with pubic pain [2]. In Hansens study however there was no difference in serum relaxin levels in patients with SPD and a control group.

Whatever the atieology and mechanism of pathopysiology, it seems to be the patients muscular reaction to the deficient pelvic stability rather than any anatomic variation that is important in causation of symptoms. This forms the basis for the use of physiotherapy in the management of the condition

\section{SYMPTOMS AND SIGNS}

The main symptom of SPD is pubic pain. This pain may range from mild to incapacitating. It is often described as 
stabbing or burning. It may be localised to the pubic bone or radiate to the lower back, groin, perineum or medial aspect of the upper thighs. The pain is often worsened by movement (particularly walking, changing position in bed, standing up from a chair, climbing or descending stairs, abduction of the hip) and relieved by rest. The pain may also be associated with a clicking sensation within the symphysis pubis which may be audible and is often quite alarming to the patient. Back, sacroiliac and hip pain may co-exist.

Signs of SPD include a waddling gait with short steps, tenderness over the pubis symphysis, tenderness over the sacro-ilac joints, a palpable gap in the symphysis pubis and a positive Trendelenburg sign on one or both sides.

Diagnosis is based on history and physical findings. Although ultrasound scan is a quick and effective way of determining interpubic gap, it is usually not employed as the width of the interpubic gap does not seem to relate to the severity of symptoms or the prognosis of SPD.

$\mathrm{X}$-ray may demonstrate the widening symphysis gap as a dark streak of gas-the so called gas phenomenon. $\mathrm{X}$-ray is not recommended as this finding does not influence management and exposes the fetus to ionising radiation.

\section{DIFFERENTIAL DIAGNOSIS}

Urinary tract infection
Diastases pubis
Oestitis pubis
Round ligament pain
Osteomyelitis

\section{INVESTIGATIONS}

Diagnosis is based on clinical symptoms and signs. Urinalysis should be performed if there are urinary symptoms and an MSSU sent for culture if urinalysis is suggestive of a urinary tract infection (UTI). In practice imaging (ultrasound, MRI, X-ray) is uneccessary.

\section{MANAGEMENT}

A clear explanation of the condition to the patient is vital as her co-operation is crucial for successful management. The psychological consequences of SPD can be quite intense and this should not be underestimated.

\subsection{Antenatal Management}

\subsubsection{Analgesia}

Simple analgesics such as paracetamol are the first choice of pain relief. This should be taken regularly for maximum effect. If these do not suffice codeine phosphate is usually commenced. NSAIDS are not advisable in pregnancy (because of fetal complications) but can be given after delivery. Opiates (morphine and tramadol) can be given as a one-off pain relief but are also not suitable for regular antenatal analgesia. Involvement of the pain relief team may be helpful.

\subsubsection{Physiotherapy}

Physiotherapy aims to correct the muscle imbalance which results from SPD. Referral to a specialist physiotherapist is advised particularly if analgesics alone are not helpful.

\subsubsection{Pelvic Support Measures}

The use of pelvic support belts (trochanteric belts) for the management of SPD has been advocated clinically and some hospitals offer these belts to patients as part of their treatment. Most of the studies that have looked at the effectiveness of belts as a pelvic stabilizing tool have however been performed on cadavers [3] (and also on sacro-illac rather than the pubic joint). Nevertheless several women are quite happy to try them.

Crutches help reduce weight bearing and are often helpful for the severely affected women. Occasionally some women become wheelchair bound as they find walking too uncomfortable.

When mobility is significantly reduced isometric exercises with the toes should be advised. Rarely (or the bed bound) heparin is given for thromboprophylaxis.

\subsubsection{Induction of Labour}

Induction of labour (after 38 weeks gestation) is often offered as a last resort if nothing else works. Ultimately delivery always seems to eventually relieve the symptoms of SPD. The potential for failed induction of labour (particularly in primiparas with an unfavourable cervix) must be discussed before induction of labour is embarked upon.

\subsection{Labour}

It is important for both midwives and doctors to be aware of the implications of SPD during labour. Separation of legs should be kept to a minimum and vaginal examinations should be peformed in a position that is most comfortable to the woman. The masking effect of epidural analgesia/spinal anaesthesia must always be remembered as it is quite easy to excessively abduct the hips if this is forgotten. Lithotomy, if recquired (e.g. for assisted delivery or suturing) should be for the shortest time possible.

\subsection{Post-Natal Management}

Following delivery analgesia should be continued as symptoms may not resolve immediately. NSAIDS can now be used. Comprehensive physiotherapy may have to be continued for a few days before symptoms eventually 
resolve. Recurrence rates in subsequent pregnancies are high.

\section{REFERENCES}

[1] Fry, D. (1999) Perinatal symphysis pubis dysfunction: A women's health review of the literature. Journal of the Association of Chartered Physiotherapists, 85, 11-17.
[2] Leadbetter, R.E., Mawer, D. and Lindow, S. (2004) Journal of Maternal-Fetal \& Neonatal medicine, 16, 349-355. doi:10.1080/jmf.16.6.349.354

[3] Depledge, J., McNair, P.J., Keal Smith, C. and Williams, M. (2005) Management of symphysis pubis dysfunction using exercises and pelvic support belts. Physical Therapy, 85, 1290-1301. 